OPEN ACCESS

Edited by: Niklas Marklund, Lund University, Sweden

Reviewed by: Hale Z. Toklu,

University of Central Florida College of Medicine, United States

Eugene Golanov,

The Houston Methodist Hospital Research Institute, United States

${ }^{*}$ Correspondence:

Aimee J. Nelson nelsonaj@mcmaster.ca

Specialty section: This article was submitted to Neurotrauma,

a section of the journal

Frontiers in Neurology

Received: 25 May 2017 Accepted: 18 July 2017 Published: 04 August 2017

Citation:

Fassett HJ, Turco CV, El-Sayes J, Lulic T, Baker S, Richardson B and

Nelson AJ (2017) Transcranial Magnetic Stimulation with Intermittent

Theta Burst Stimulation Alters

Corticospinal Output in Patients with Chronic Incomplete Spinal Cord Injury.

Front. Neurol. 8:380

doi: 10.3389/fneur.2017.00380

\section{Transcranial Magnetic Stimulation with Intermittent Theta Burst Stimulation Alters Corticospinal Output in Patients with Chronic Incomplete Spinal Cord Injury}

\author{
Hunter J. Fassett', Claudia V. Turco ${ }^{1}$, Jenin El-Sayes ${ }^{1}$, Tea Lulic ${ }^{1}$, Steve Baker ${ }^{2}$, \\ Brian Richardson ${ }^{1}$ and Aimee J. Nelson ${ }^{1 *}$
}

${ }^{1}$ Department of Kinesiology, McMaster University, Hamilton, ON, Canada, ${ }^{2}$ Division of Physical Medicine and Rehabilitation, Department of Medicine, McMaster University, Hamilton, ON, Canada

Intermittent theta burst stimulation (iTBS) is intended primarily to alter corticospinal excitability, creating an attractive opportunity to alter neural output following incomplete spinal cord injury (SCl). This study is the first to assess the effects of iTBS in SCl. Eight individuals with chronic incomplete SCI were studied. Sham or real iTBS was delivered (to each participant) over primary motor and somatosensory cortices in separate sessions. Motor-evoked potential (MEP) recruitment curves were obtained from the flexor carpi radialis muscle before and after iTBS. Results indicate similar responses for iTBS to both motor and somatosensory cortex and reduced MEPs in $56.25 \%$ and increased MEPs in 25\% of instances. Sham stimulation exceeded real iTBS effects in the remaining $18.25 \%$. It is our opinion that observing short-term neuroplasticity in corticospinal output in chronic $\mathrm{SCl}$ is an important advance and should be tested in future studies as an opportunity to improve function in this population. We emphasize the need to re-consider the importance of the direction of MEP change following a single session of iTBS since the relationship between MEP direction and motor function is unknown and multiple sessions of iTBS may yield very different directional results. Furthermore, we highlight the importance of including sham control in the experimental design. The fundamental point from this pilot research is that a single session of iTBS is often capable of creating shortterm change in SCI. Future sham-controlled randomized trials may consider repeat iTBS sessions to promote long-term changes in corticospinal excitability.

Keywords: neuroplasticity, transcranial magnetic stimulation, spinal cord injury, placebo, sensorimotor cortex, TBS

\section{INTRODUCTION}

Following spinal cord injury (SCI), damage to the ascending and descending spinal pathways leads to sensory and motor impairments below the level of injury. Opportunities to recapture or improve neural output to impaired muscles are of utmost importance, particularly for muscles of the upper limb to allow for greater independence performing activities of daily living. Repetitive transcranial magnetic stimulation (TMS) may provide an opportunity to promote motor recovery 
in incomplete SCI by strengthening synaptic connectivity within intact descending fibers thereby increasing neural output to affected muscles. To date, repetitive TMS protocols have shown mixed results when assessing recovery of function following SCI $(1,2)$ with only one report showing increases in corticospinal output (3). Spinal associative plasticity that pairs TMS and nerve stimulation increases neural output to muscles of the hand and improves function $(4,5)$.

Patterned, rapid delivery of TMS is delivered in a protocol called intermittent theta burst stimulation (iTBS). iTBS delivered over primary motor cortex (M1) facilitates corticospinal output as measured by increases in the amplitude of motor-evoked potentials (MEPs) (6-8) but is also equally effective at reducing MEPs (7). Therefore, caution should be taken when assuming that iTBS should evoke a particular directional effect. Further, there is no clear relationship between the direction of iTBS-induced changes in MEPs and predictable change in motor behavior. However, to date, the evidence suggests that iTBS does indeed alter corticospinal output in healthy, uninjured controls. The advantage of iTBS relative to other TMS approaches is the short duration required to deliver the protocol $(\sim 2 \mathrm{~min})$ and its low intensity, making it attractive for both experimenters and participants. To date, no study has examined the effects of iTBS in individuals with incomplete SCI, yet this technique has the potential to alter corticospinal output to impaired muscles of the arm.
We present a sham-controlled pilot study to provide the first characterization of iTBS-induced effects in chronic, incomplete cervical SCI. We consider these pilot data timely since recent SCI research in rodents indicates that repeat sessions of iTBS lead to facilitation of MEPs (9). Before repeat iTBS is delivered in humans with SCI, it is important to demonstrate whether a single session of iTBS is capable of inducing short-term changes as observed in uninjured controls $(7,10-12)$. To test this, we delivered real and sham iTBS over M1, and real iTBS was delivered over primary somatosensory cortex (S1). We included S1 as a novel target since iTBS targeting $\mathrm{S} 1$ has primarily shown facilitation of sensory physiology (13-15) and improvement of sensory discrimination $(15,16)$. Given that neuroplasticity involving reorganization in SCI likely occurs in both M1 and $S 1$, we considered that stimulation over either area may influence the excitability of adjacent M1 to ultimately alter the neural output to the target muscles.

\section{METHODS}

Eight individuals with chronic ( $>1$ year post-injury) incomplete cervical (injury from C4-T1, ASIA classification as B, C, or D) SCI participated (Table 1) in three experimental sessions. Subjects were screened for contraindications to TMS prior to participation. The study conformed to the declaration of Helsinki and was

TABLE 1 | Participant demographics and results.

\begin{tabular}{|c|c|c|c|c|c|c|c|c|}
\hline ID & Age & Gender & Injury level & Years post-injury & ASIA & Medications & iTBS site & MEP \\
\hline 1 & 29 & $M$ & C4 & 5 & C & Baclofen & $\begin{array}{l}\text { M1 } \\
\text { S1 }\end{array}$ & $\begin{array}{c}\downarrow(21.8 \%) \\
\text { Sham > real }\end{array}$ \\
\hline 2 & 39 & $M$ & $\mathrm{C} 6-\mathrm{C} 7$ & 14 & C & Baclofen & $\begin{array}{l}\text { M1 } \\
\text { S1 }\end{array}$ & $\begin{array}{l}\uparrow(4.0 \%) \\
\downarrow(20.3 \%)\end{array}$ \\
\hline 3 & 26 & $M$ & C5 & 4.5 & C & Fesoterodine & $\begin{array}{l}\text { M1 } \\
\text { S1 }\end{array}$ & $\begin{array}{l}\downarrow(30.8 \%) \\
\downarrow(26.7 \%) \\
\end{array}$ \\
\hline 4 & 41 & $M$ & C6 & 2 & $\mathrm{D}$ & $\begin{array}{l}\text { Diazepam, pregabalin, } \\
\text { cyclobenzaprine }\end{array}$ & $\begin{array}{l}\text { M1 } \\
\text { S1 }\end{array}$ & $\begin{array}{c}\text { Sham }>\text { real } \\
\downarrow(15.9 \%)\end{array}$ \\
\hline 5 & 39 & M & C5 & 39 & N/A & None & $\begin{array}{l}\text { M1 } \\
\text { S1 }\end{array}$ & $\begin{array}{l}\uparrow(73.9 \%) \\
\downarrow(25.3 \%) \\
\end{array}$ \\
\hline 6 & 55 & $\mathrm{~F}$ & C3-C4 & 2 & C & Gabapentin, citalopram & $\begin{array}{l}\text { M1 } \\
\text { S1 }\end{array}$ & $\begin{array}{c}\text { Sham > real } \\
\uparrow(20.9 \%) \\
\end{array}$ \\
\hline 7 & 58 & $M$ & $\mathrm{C} 6-\mathrm{C} 7$ & 33 & B & Baclofen, clonazepam & $\begin{array}{l}\text { M1 } \\
\text { S1 }\end{array}$ & $\begin{array}{l}\downarrow(55.0 \%) \\
\uparrow(13.1 \%) \\
\end{array}$ \\
\hline 8 & 68 & $M$ & C4 & 3 & B & Baclofen, pregabalin & $\begin{array}{l}\text { M1 } \\
\text { S1 }\end{array}$ & $\begin{array}{l}\downarrow(3.9 \%) \\
\downarrow(21.4 \%)\end{array}$ \\
\hline & & & & & & \multicolumn{2}{|c|}{ Percent of instances demonstrating change $(N=16)$} & MEP \\
\hline & & & & & & \multicolumn{2}{|c|}{$\uparrow$} & $25 \%$ \\
\hline & & & & & & \multicolumn{2}{|c|}{$\downarrow$} & $56.25 \%$ \\
\hline & & & & & & \multicolumn{2}{|c|}{ Sham > Real } & $18.75 \%$ \\
\hline
\end{tabular}

Participant demographic information and individual responses to iTBS are shown.

M, male; F, female; C, cervical spine (i.e., C4); iTBS, intermittent theta burst stimulation; M1, primary motor cortex; S1, primary somatosensory cortex; ASIA, American Spinal Injury Association Impairment Scale $(A=$ no sensory or motor function preserved in sacral segments; $B=$ sensory function is preserved with no motor function; $C=$ sensory function is preserved below the level of injury, most muscles below injury have a grade less than 3; D = motor function is preserved below the level of injury, most muscles below injury have a grade of 3 or more; $E=$ normal sensory and motor function); RMT, resting motor threshold; MEP, motor-evoked potential; $\uparrow$, measure is increased following iTBS relative to sham effect (percentage of change exceeding sham effects); $\downarrow$, measure is decreased following iTBS relative to sham effect (percentage of change exceeding sham effects); $N / A$, data could not be obtained. 
approved by the Hamilton Integrated Research Ethics Board. All individuals provided written consent prior to participation.

Electromyography (EMG) was recorded using surface electrodes ( $9 \mathrm{~mm}$ diameter $\mathrm{Ag}-\mathrm{AgCl}$ ) that were placed in a belly-belly montage on the flexor carpi radialis (FCR) and extensor carpi radialis muscles with a wet ground placed around the forearm proximal to the recording electrodes (Figure 1). All EMG recordings were band-pass filtered between $20 \mathrm{~Hz}$ and $2.5 \mathrm{kHz}$, amplified 1,000× (Intronix Technologies Corporation Model 2024F, Bolton, Canada), and digitized using an analog-to-digital interface at $5 \mathrm{kHz}$ (Power1401, Cambridge Electronics Design, Cambridge, UK). Data were collected using Signal software (v6.02, Cambridge Electronics Design, Cambridge, UK).

In this study, iTBS was delivered over the hemisphere contralateral to the least impaired FCR muscle. To determine the least impaired FCR, maximum voluntary contraction was recorded in three trials from the FCR muscle while maintaining a maximum isometric contraction against an immovable post. Each trial consisted of MVC for $5 \mathrm{~s}$ followed by $1 \mathrm{~min}$ of rest. Visual feedback from an oscilloscope (Tektronix TDS2004c, USA) displayed muscle activity to the participant. The maximum peak-to-peak amplitude achieved across all trials was documented as the MVC. The FCR that produced the greatest MVC was taken as the least impaired limb.

Single-pulse TMS was delivered via a $50 \mathrm{~mm}$ diameter figureof-eight branding coil connected to a Magstim $200^{2}$ stimulator (Magstim, UK) over the optimal location (i.e., motor hotspot) to elicit MEPs in the relaxed FCR of the least affected arm. The coil was positioned $45^{\circ}$ in relation to the parasagittal plane to induce posterior-to-anterior current in the cortex. The motor hotspot was marked by digital registration using a standard MRI template via Brainsight Neuronavigation (Rogue Research, Canada). At this location, resting motor threshold (RMT) was quantified as the percentage of maximum stimulator output that elicited MEPs $\geq 50 \mu \mathrm{V}$ peak-to-peak amplitude in 5 out of 10 consecutive trials (17). Active motor threshold (AMT) was determined as the percentage of maximum stimulator output that produced an MEP of $\geq 200 \mu \mathrm{V}$ peak-to-peak amplitude in 5 out of 10 consecutive trials while participants maintained a contraction of $15 \%$ of their MVC.
Intermittent theta burst stimulation protocol was delivered using a $70 \mathrm{~mm}$ inner diameter figure-of-eight coil with a Magstim Super Rapid ${ }^{2}$ Plus (Magstim, Whitland, UK) using biphasic pulses in bursts of three pulses delivered at $30 \mathrm{~Hz}$, in $6 \mathrm{~Hz}$ trains that lasted $2 \mathrm{~s}$, that was followed by a period of $8 \mathrm{~s}$ in which no pulses were delivered (8). iTBS was repeated for a total of 612 pulses delivered at $80 \%$ AMT. Participants received one of three iTBS interventions in each session and the order of delivery was pseudorandomized across participants: iTBS-M1 delivered at the motor hotspot for the least affected FCR, iTBS-S1 delivered at a position digitally marked $2 \mathrm{~cm}$ posterior to the FCR motor hotspot, and sham iTBS delivered over the FCR motor hotspot. This sham coil appears and sounds like verum iTBS but does not deliver real pulses. All TBS (including real and sham) was delivered to the hemisphere contralateral to the target FCR muscle (i.e., the least affected).

Motor-evoked potential recruitment curves were recorded before and immediately following the iTBS intervention in each session. Single-pulse TMS was applied over the FCR motor hotspot at 8 different stimulus intensities: 90, 100, 110, 120, 130, 140,150 , and $160 \%$ of RMT. Three pulses were delivered at each intensity in a randomized order. The average area of the MEP was quantified at each intensity by identifying the area under the $\mathrm{MEP}$ in each trial within a $30 \mathrm{~ms}$ window. The area of the MEP was measured.

To assess the effects of iTBS across the entire recruitment curve, we quantified the area under the MEP recruitment curve (AURC) for each participant as this has been shown to be a reliable way to assess corticospinal excitability changes in proximal upper arm muscles (18). For MEP recruitment curves, we calculated the percent change from $\mathrm{T} 0$ to $\mathrm{T} 1$ following real iTBS to either M1 or S1 and subtracted the percent change from T0 to T1 obtained from the sham condition. Therefore, on a case-by-case basis, we only considered an effect to be "real" if it exceeded the effect of sham, or if it was in the direction opposite to sham effects (Eq. 1).

$$
\% \text { Change }=100 \times\left(\frac{\mathrm{T} 1_{\text {real }}}{\mathrm{T} 0_{\text {real }}}-1\right)-100 \times\left(\frac{\mathrm{T} 1_{\text {sham }}}{\mathrm{T} 0_{\text {sham }}}-1\right)
$$
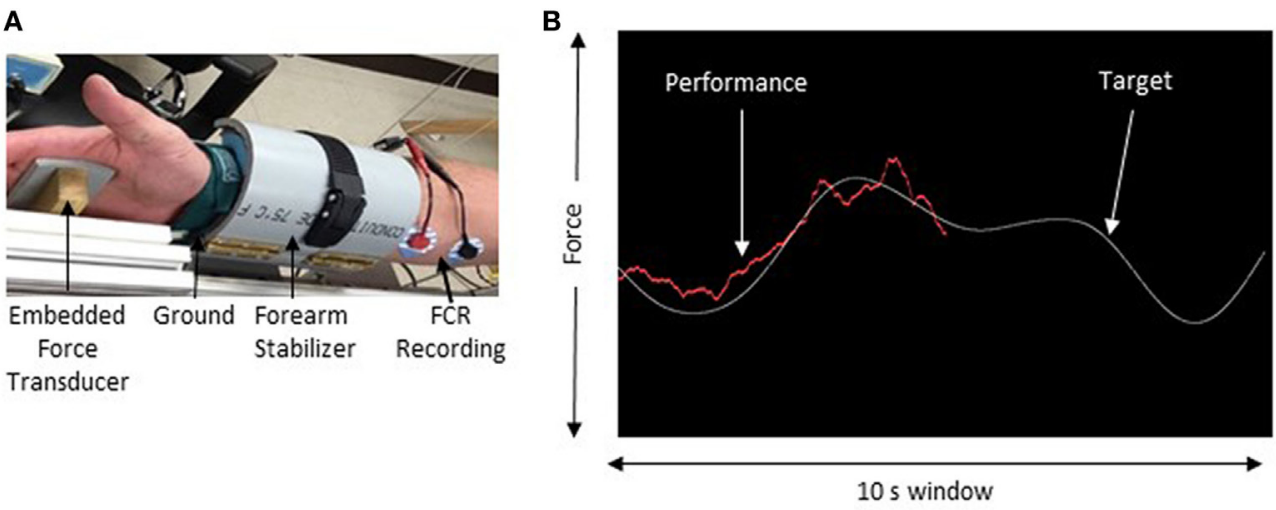

FIGURE 1 | Experimental setup. (A) The apparatus used to maintain forearm position during force tracking. (B) Sample of the force tracking task. The white line represents the target waveform and the red line is controlled by the force transducer and represents the participant's performance. 


\section{RESULTS}

The effects of real and sham iTBS are shown in Table $\mathbf{1}$ for each participant. We observed no differences associated with the location of stimulation (S1 versus M1); MEPs decreased in 50 and $62.5 \%$ of subjects for M1 and S1, respectively, and increased in $25 \%$ of participants for both M1 and S1. In total, following subtraction of sham effects, iTBS decreased MEPs in $56.25 \%$ of instances (9/16) and increased MEPs in 25\% of instances. Real iTBS did not exceed the effects of sham in the remaining $18.75 \%$ (Table 1).

\section{DISCUSSION}

The data demonstrate that iTBS over M1 and/or S1 led to changes in MEPs in $81.25 \%$ of instances, and these were changes that exceeded those induced by sham stimulation. The present data from eight individuals with chronic SCI indicate that a single session of iTBS over M1 or the adjacent SI tends to decrease MEPs in FCR. These data are promising since they indicate that corticospinal excitability is modifiable in this population. We note that sham effects exceeded real iTBS effects in 20\% of instances and this finding highlights the importance of including sham control stimulation in future studies. One limitation is the lack of imaging data to quantify the spinal cord lesion characteristics in our participants.

Previous research suggests that iTBS should be expected to increase MEPs (7). However, in a larger sample study, iTBS was shown to suppress MEPs in $48 \%$ and increase in 52\%, leading to nearly proportional outcomes (10). Further, it is unclear whether increases or decreases in MEPs have direct relationships with motor performance from a given muscle. Therefore, in the present study, our focus was not on the direction of change induced by iTBS but rather whether MEPs were indeed modifiable with iTBS in chronic SCI. Our data from our sample of individuals indicate that corticospinal excitability to an impaired muscle in SCI is modifiable, and this information is important since iTBS is of minimal imposition to a participant requiring low-intensity stimulation for less than $3 \mathrm{~min}$. Work in other clinical populations has shown that iTBS may not be as effective. Notably, iTBS does not alter MEPs in Tourette's syndrome $(19,20)$, focal hand

\section{REFERENCES}

1. Belci M, Catley M, Husain M, Frankel HL, Davey NJ. Magnetic brain stimulation can improve clinical outcome in incomplete spinal cord injured patients. Spinal Cord (2004) 42:417-9. doi:10.1038/s.sc.3101613

2. Benito J, Kumru H, Murillo N, Costa U, Medina J, Tormos J, et al. Motor and gait improvement in patients with incomplete spinal cord injury induced by high-frequency repetitive transcranial magnetic stimulation. Top Spinal Cord Inj Rehabil (2012) 18:106-12. doi:10.1310/sci1802-106

3. Alexeeva N, Calancie B. Efficacy of QuadroPulse rTMS for improving motor function after spinal cord injury: three case studies. J Spinal Cord Med (2016) 39:50-7. doi:10.1179/2045772314Y.0000000279

4. Bunday KL, Perez MA. Motor recovery after spinal cord injury enhanced by strengthening corticospinal synaptic transmission. Curr Biol (2012) 22:2355-61. doi:10.1016/j.cub.2012.10.046 dystonia (21), multiple system atrophy (22), Alzheimer's disease (23), and Parkinson's disease (24). However, another study demonstrated suppression of MEPs in individuals with Parkinson's diseases when iTBS is delivered to the more affected hemisphere (25). The data from this pilot study indicate that iTBS may provide an opportunity for inducing changes in corticospinal excitability, in line with recent iTBS study in rodent model (9), albeit we did not typically see facilitation. This may relate to the single versus multiple trains of iTBS between the studies, or differences across species.

We conclude that a single session of iTBS tends to decrease MEPs in individuals with incomplete chronic SCI. Our findings highlight the need to reevaluate our expectation of the effects of a single session of iTBS effects in SCI as we observed suppression more frequently than facilitation following a single bout of stimulation. It is our opinion that these pilot data are promising since they suggest that changes in corticospinal excitability are possible in chronic SCI, which creates an avenue for future research in this population. These data may assist with the development and design of future, larger-scale studies regarding the anticipated effects of iTBS in chronic SCI.

\section{ETHICS STATEMENT}

The study conformed to the declaration of Helsinki and was approved by the Hamilton Integrated Research Ethics Board.

\section{AUTHOR CONTRIBUTIONS}

HF conceived of the study, collected, analyzed, and interpreted data, and drafted the manuscript. CT and JE-S collected, analyzed, and interpreted data and edited manuscript. TL collected data and edited manuscript. BR designed technical aspects and edited manuscript. SB provided oversight on SCI participants and edited manuscript. AN conceived of the study, analyzed and interpreted data, and drafted the manuscript.

\section{FUNDING}

The authors thank CRC funding to AJN.

5. Urbin MA, Ozdemir RA, Tazoe T, Perez MA. Spike-timing-dependent plasticity in lower-limb motoneurons after human spinal cord injury. J Neurophysiol (2017). doi:10.1152/jn.00111.2017

6. Hinder MR, Goss EL, Fujiyama H, Canty AJ, Garry MI, Rodger J, et al. Interand intra-individual variability following intermittent theta burst stimulation: implications for rehabilitation and recovery. Brain Stimulat (2014) 7:365-71. doi:10.1016/j.brs.2014.01.004

7. Huang Y-Z, Edwards MJ, Rounis E, Bhatia KP, Rothwell JC. Theta burst stimulation of the human motor cortex. Neuron (2005) 45:201-6. doi:10.1016/j. neuron.2004.12.033

8. Wu SW, Shahana N, Huddleston DA, Gilbert DL. Effects of $30 \mathrm{~Hz}$ theta burst transcranial magnetic stimulation on the primary motor cortex. J Neurosci Methods (2012) 208:161-4. doi:10.1016/j.jneumeth.2012.05.014

9. Song W, Amer A, Ryan D, Martin JH. Combined motor cortex and spinal cord neuromodulation promotes corticospinal system functional and structural 
plasticity and motor function after injury. Exp Neurol (2016) 277:46-57. doi:10.1016/j.expneurol.2015.12.008

10. Hamada M, Murase N, Hasan A, Balaratnam M, Rothwell JC. The role of interneuron networks in driving human motor cortical plasticity. Cereb Cortex (2013) 23:1593-605. doi:10.1093/cercor/bhs147

11. Jacobs MF, Tsang P, Lee KGH, Asmussen MJ, Zapallow CM, Nelson AJ. $30 \mathrm{~Hz}$ theta-burst stimulation over primary somatosensory cortex modulates corticospinal output to the hand. Brain Stimulat (2014) 7:269-74. doi:10.1016/j. brs.2013.12.009

12. Jacobs MF, Zapallow CM, Tsang P, Lee KGH, Asmussen MJ, Nelson AJ. Current direction specificity of continuous theta-burst stimulation in modulating human motor cortex excitability when applied to somatosensory cortex. Neuroreport (2012) 23:927-31. doi:10.1097/WNR.0b013e328358b0f3

13. Katayama T, Rothwell JC. Modulation of somatosensory evoked potentials using transcranial magnetic intermittent theta burst stimulation. Neurophysiol Clin (2007) 118:2506-11. doi:10.1016/j.clinph.2007.08.011

14. Katayama T, Suppa A, Rothwell JC. Somatosensory evoked potentials and high frequency oscillations are differently modulated by theta burst stimulation over primary somatosensory cortex in humans. Neurophysiol Clin (2010) 121:2097-103. doi:10.1016/j.clinph.2010.05.014

15. Ragert P, Franzkowiak S, Schwenkreis P, Tegenthoff M, Dinse HR. Improvement of tactile perception and enhancement of cortical excitability through intermittent theta burst rTMS over human primary somatosensory cortex. Exp Brain Res (2007) 184:1-11. doi:10.1007/s00221-007-1073-2

16. Conte A, Rocchi L, Nardella A, Dispenza S, Scontrini A, Khan N, et al. Thetaburst stimulation-induced plasticity over primary somatosensory cortex changes somatosensory temporal discrimination in healthy humans. PLoS One (2012) 7:e32979. doi:10.1371/journal.pone.0032979

17. Rossini PM, Burke D, Chen R, Cohen LG, Daskalakis Z, Di Iorio R, et al. Non-invasive electrical and magnetic stimulation of the brain, spinal cord, roots and peripheral nerves: basic principles and procedures for routine clinical and research application. An updated report from an I.F.C.N. Committee. Clin Neurophysiol (2015) 126:1071-107. doi:10.1016/j. clinph.2015.02.001

18. Carson RG, Nelson BD, Buick AR, Carroll TJ, Kennedy NC, Cann RM. Characterizing changes in the excitability of corticospinal projections to proximal muscles of the upper limb. Brain Stimulat (2013) 6:760-8. doi:10.1016/j.brs.2013.01.016

19. Suppa A, Belvisi D, Bologna M, Marsili L, Berardelli I, Moretti G, et al. Abnormal cortical and brain stem plasticity in Gilles de la Tourette syndrome. Mov Disord (2011) 26:1703-10. doi:10.1002/mds.23706

20. Wu SW, Gilbert DL. Altered neurophysiologic response to intermittent theta burst stimulation in Tourette syndrome. Brain Stimul (2012) 5:315-9. doi:10.1016/j.brs.2011.04.001

21. Belvisi D, Suppa A, Marsili L, Di Stasio F, Parvez AK, Agostino R, et al. Abnormal experimentally- and behaviorally-induced LTP-like plasticity in focal hand dystonia. Exp Neurol (2013) 240:64-74. doi:10.1016/j.expneurol.2012.11.003

22. Suppa A, Marsili L, Di Stasio F, Latorre A, Parvez AK, Colosimo C, et al. Primary motor cortex long-term plasticity in multiple system atrophy. Mov Disord (2014) 29:97-104. doi:10.1002/mds.25668

23. Koch G, Di Lorenzo F, Bonnì S, Ponzo V, Caltagirone C, Martorana A. Impaired LTP- but not LTD-like cortical plasticity in Alzheimer's disease patients. J Alzheimers Dis (2012) 31:593-9. doi:10.3233/JAD-2012-120532

24. Suppa A, Marsili L, Belvisi D, Conte A, Iezzi E, Modugno N, et al. Lack of LTP-like plasticity in primary motor cortex in Parkinson's disease. Exp Neurol (2011) 227:296-301. doi:10.1016/j.expneurol.2010.11.020

25. Kishore A, Joseph T, Velayudhan B, Popa T, Meunier S. Early, severe and bilateral loss of LTP and LTD-like plasticity in motor cortex (M1) in de novo Parkinson's disease. Clin Neurophysiol (2012) 123:822-8. doi:10.1016/j. clinph.2011.06.034

Conflict of Interest Statement: The authors declare that the research was conducted in the absence of any commercial or financial relationships that could be construed as a potential conflict of interest.

Copyright (c) 2017 Fassett, Turco, El-Sayes, Lulic, Baker, Richardson and Nelson. This is an open-access article distributed under the terms of the Creative Commons Attribution License (CC BY). The use, distribution or reproduction in other forums is permitted, provided the original author(s) or licensor are credited and that the original publication in this journal is cited, in accordance with accepted academic practice. No use, distribution or reproduction is permitted which does not comply with these terms. 